\title{
Cognitive dysfunction in adults with Van der Woude syndrome
}

Peg Nopoulos, $M D^{1}$, Lynn Richman, $P h D^{2}$, Nancy Andreasen, $M D, P h D^{2}$, Jeffrey C. Murray, $M D^{2}$, and Brian Schutte, $P h D^{2}$

\begin{abstract}
Purpose: Van der Woude syndrome is an autosomal dominant disorder manifested in clefts of the lip and/or palate and lip pits. There is phenotypic and genotypic overlap between Van der Woude syndrome and isolated cleft of the lip and/or palate. Subjects with isolated cleft of the lip and/or palate have been shown to have cognitive dysfunction. Given the similarities between Van der Woude syndrome and isolated cleft of the lip and/or palate, the current study was designed to evaluate the pattern of cognitive function in adults with Van der Woude syndrome. Methods: Fourteen adults with Van der Woude syndrome were compared with age- and gender-matched controls. A battery of cognitive tests was administered to determine general IQ as well as more specific cognitive performance measures. Results: All subjects with Van der Woude syndrome showed deficits in performance on an executive function task. In addition, males with Van der Woude syndrome had significantly lower scores on intelligence measures and on a verbal fluency task compared with controls. Conclusion: The pattern of cognitive function in Van der Woude syndrome is very similar to that seen in isolated cleft of the lip and/or palate. In addition, the findings have a significant gender effect in which males are more severely affected than females. This pattern is common to other conditions with a neurodevelopmental etiology, supporting the notion that the cognitive deficits of Van der Woude syndrome and isolated cleft of the lip and/or palate are due to abnormal brain development.
\end{abstract}

Genet Med 2007:9(4):213-218.

Key Words: Van der Woude syndrome, Cleft lip and/or palate, Cognitive function

Van der Woude syndrome (VWS; OMIM 119300) is an orofacial clefting disorder with an autosomal dominant pattern of inheritance. In 2002, Kondo et al. ${ }^{1}$ reported that mutations in interferon regulatory factor 6 (IRF6) cause VWS. The cardinal features of VWS include cleft lip with or without cleft palate and lip pits. Associated abnormalities include hypodontia ${ }^{2,3}$ and limb, skin, nail, and genital abnormalities or hearing loss. ${ }^{4}$ The phenotypic manifestations of VWS are very similar to that of nonsyndromic or isolated clefts of the lip and/or palate (ICLP). In addition, a DNA sequence variation in IRF6 confers a risk for isolated cases of cleft lip and palate. ${ }^{5}$ Thus, there is not only phenotype, but genotype overlap between VWS and ICLP.

School-age children with ICLP have consistently been reported to have cognitive dysfunction that is characterized by a significant lowering of full scale IQ (FSIQ), and specific deficits in expressive language. ${ }^{6,7} \mathrm{In}$ a previous study in our lab, adult males with ICLP were found to have a FSIQ in the average

From the Departments of ${ }^{1}$ Psychiatry and ${ }^{2}$ Pediatrics, University of Iowa College of Medicine, Iowa City, Iowa.

Peg Nopoulos, Department of Psychiatry, Iowa Neuroimaging Center, University of Iowa College of Medicine, 200 Hawkins Drive, W278 GH, Iowa City, IA 52242; E-mail: peggy-nopoulos@ uiowa.edu

The authors declare no conflict of interest.

Submitted for publication October 4, 2006.

Accepted for publication January 4, 2007.

DOI: 10.1097/GIM.0b013e3180335abd range, but significantly lower than a group of matched healthy controls. ${ }^{8}$ Moreover, after controlling for FSIQ, this group manifested specific deficits in verbal fluency with less significant deficits in frontal lobe tasks (set-shifting) and verbal memory. Despite the body of work showing cognitive deficits in ICLP, the cognitive function of subjects with VWS has never before been reported.

The current study was designed to evaluate brain function using performance on cognitive tests in a group of adults with VWS compared with healthy controls. Due to the substantial overlap in phenotype and genotype between VWS and ICLP, we hypothesize that individuals with VWS will manifest the same pattern of cognitive abnormalities as that seen in ICLP. A secondary hypothesis was regarding gender effects. All neurodevelopmental cognitive disorders, in particular those based in language development, are more common and more severe in males. ${ }^{9,10}$ Our previous study of adults with ICLP was limited to a male sample, and our hypothesis for the current study was that the females with VWS would manifest the same pattern of cognitive deficits as the males, but that their deficits would be less severe compared with the males with VWS.

\section{METHODS}

\section{Participants}

Families with VWS were identified and examined by one or more geneticists or clinicians, establishing a database of poten- 
tial subjects. Families were ascertained through contact with geneticists, orthodontists, or cleft palate clinics. Individuals were considered to be affected with VWS if they had one or more of cleft lip, cleft palate, hypodontia, or lower-lip pits with at least one individual in the pedigree having lip pits. All the families had multiple affected individuals. We identified those who were older than 18 and located within 500 miles to come to the hospital and participate in our protocol. A total of seven males and seven females with VWS agreed to participate. The entire VWS sample represented eight families with seven subjects all belonging to one large extended family and the remaining seven subjects coming from seven different families. The protocol for this study was approved by the University Institutional Review Board. All subjects signed informed consent before study enrollment.

Control subjects were selected from a registry of healthy volunteers with neuropsychological data and accompanying magnetic resonance imaging brain scan collected via the Mental Health Clinical Research Center. These subjects were recruited through newspaper advertising and screened with a structured interview. Control subjects were excluded if they had a significant medical (including cleft lip and/or palate), neurological or psychiatric illness, including alcohol and/or substance abuse. Controls were matched to subjects by age (within 2 years) and sex. Reflective of the population in Iowa, all the subjects and controls were white. Education level (in years) of all subjects as well as education level (in years) for parents (mother + father) was also documented.

Demographics of the study groups are shown in Table 1. The education level of the subjects was lower than those of the controls. Using paired $t$ tests, this difference was nonsignificant $(t=1.7, P=0.09)$. Parental education is an appropriate proxy for social class. Factors associated with variances in social class may potentially affect brain growth and development, such as nutrition, infections, and access to medical care. The subjects in our study had a parental education level that was lower than those of the controls, although this difference was nonsignificant $(t=1.9, P=0.06)$.

The phenotype of VWS subjects is shown in Table 2. All subjects had lip pits. Four subjects had clefts of the lip and palate and six subjects had cleft palate only. Four subjects had no oral clefting and manifested lip pits only.

Table 1

Demographics

\begin{tabular}{lcccr}
\hline & $\begin{array}{c}\text { VWS subjects, } \\
\text { mean (SD) } \\
(n=14)\end{array}$ & $\begin{array}{c}\text { Controls, } \\
\text { mean (SD) } \\
(n=14)\end{array}$ & $t$ & $P$ \\
\hline Age, yr & $34.0(9.0)$ & $33.3(7.1)$ & 0.23 & 0.81 \\
Education, yr & $12.7(1.9)$ & $14.0(1.7)$ & 1.72 & 0.09 \\
Parental education, yr & $23.3(3.3)$ & $25.3(2.7)$ & 1.91 & 0.06 \\
Male/female & $7 / 7$ & $7 / 7$ & N/A & \\
\hline
\end{tabular}

VWS, Van der Woude syndrome.
Table 2

VWS phenotype

\begin{tabular}{lcccc}
\hline & $\begin{array}{c}\text { Bilateral } \\
\text { cleft lip } \\
\text { and palate }\end{array}$ & $\begin{array}{c}\text { Unilateral } \\
\text { cleft lip } \\
\text { and palate }\end{array}$ & $\begin{array}{c}\text { Cleft palate } \\
\text { only }\end{array}$ & $\begin{array}{c}\text { Lip pits } \\
\text { only }\end{array}$ \\
\hline Males & 2 & 1 & 2 & 2 \\
Females & 1 & 0 & 4 & 2 \\
\hline
\end{tabular}

VWS, Van der Woude syndrome.

\section{Cognitive testing}

For testing of general intelligence, the Wechsler Adult Intelligence Scale-Revised ${ }^{11}$ (WAIS-R) was used. Variables used included FSIQ, Performance IQ (PIQ) and Verbal IQ (VIQ).

Additional tests included a language task: Word Fluency Test [number of words generated in 1 minute in response to three different letters (C, F, and L) $]^{12}$ and a test of verbal memory, the Rey Auditory-Verbal Learning Test (RAVLT, immediate recall and delayed recall). ${ }^{13}$ Visual perceptual skills were tested using the Rey-Osterreith Complex Figure Test (copy, immediate recall, and delayed recall). ${ }^{14,15}$ Executive function was assessed with the Stroop Test (interference score). ${ }^{16}$

\section{Statistical analysis}

All analyses were performed by using the SAS language with SAS STAT procedures. To compare IQ scores across groups, analysis of covariance (ANCOVA) was used. Although the parental education level was not statistically different between the two groups, this variable is a potential confound and therefore was controlled for by including it as the covariate in this analysis. To investigate the role of gender, the variable of sex was also entered into the model. For all comparisons of the additional cognitive tests, ANCOVA was used with sex and FSIQ as the covariates. All possible interaction terms were entered into the model, but dropped if not significant. An $\alpha$ level of 0.05 was used for significance tests, except for tests for assumptions within the general linear model, in which case an $\alpha$ of 0.10 was used. That is, if an interaction term was found to be significant at $P<0.10$, it was maintained within the model. For those measures in which there was a significant sex by diagnosis interaction, follow-up analysis was done using a post hoc $t$ test, breaking the groups down by gender.

\section{RESULTS}

Table 3 shows the adjusted means and SDs of each IQ test for both groups. The subjects with VWS had significantly lower FSIQ and VIQ scores compared with the controls. There was no difference between groups in the PIQ score. Figure 1 shows the distribution of FSIQ between the two groups. The normal control group has a distribution with IQs ranging from 96 to 123. The VWS group has a slightly wider distribution with a range of 84 to 118 . However, the VWS group clearly has a shift downward in distribution with disproportionate numbers of sub- 
Table 3

Adjusted means, SDs, and analysis results of IQ tests

\begin{tabular}{lccccccc}
\hline & $\begin{array}{c}\text { Subjects } \\
\text { with VWS }\end{array}$ & $\begin{array}{c}\text { Healthy } \\
\text { controls }\end{array}$ & \multicolumn{2}{c}{$\begin{array}{c}\text { Effect of } \\
\text { diagnosis }\end{array}$} & \multicolumn{2}{c}{$\begin{array}{c}\text { Sex by diagnosis } \\
\text { interaction }\end{array}$} \\
\hline FSIQ & $97.3(8.31)$ & $106.3(7.81)$ & 9.28 & 0.005 & 6.71 & 0.016 \\
VIQ & $95.1(9.17)$ & $104.2(10.25)$ & 6.75 & 0.016 & 7.99 & 0.009 \\
PIQ & $102.2(9.36)$ & $109.0(11.42)$ & 2.60 & 0.120 & 0.19 & 0.663 \\
\hline
\end{tabular}

VWS, Van der Woude syndrome; FSIQ, full-scale IQ; VIQ, Verbal IQ; PIQ, Performance IQ.

jects in the lower tertile of the range. Although the control group has mean IQs above average, these levels are typical for the Iowa population, which generally has an average IQ around $110 .{ }^{17}$

Table 4 shows the analysis of the IQ scores broken down by group and gender. There was no main effect of sex on FSIQ $(F=$ $0.39, P=0.538)$, VIQ $(F=1.46, P=0.238)$, or PIQ $(F=0.55$, $P=0465)$. However, there were significant sex by diagnosis interactions for both FSIQ $(F=6.71, P=0.016)$ and VIQ measures $(F=7.99, P=0.009)$. Comparative groups in this analysis are now quite small $(n=7)$, so results should be viewed with caution. These findings show that the main effect of diagnosis on FSIQ and VIQ is accounted for by the males with VWS.

After controlling for FSIQ, there was a main effect of sex on the Stroop Test interference score $(F=16.6, P=0.005)$ and the test of verbal memory (RAVLT), both the immediate $(F=$ 5.67, $P=0.026)$ and delayed scores $(F=11.23, P=0.003)$. In all three of these tests, all females scored significantly higher than the males: Stroop Test-adjusted mean for males $=41.4$; adjusted mean for females $=52.3$; RAVLT immediate-adjusted mean for males $=52.6$, adjusted mean for females $=$ 62.0; RAVLT delayed-adjusted mean for males $=7.30$, adjusted mean for females $=10.49$.

Table 5 shows the results of the additional cognitive tests compared across the two study groups. After controlling for FSIQ and gender, there was a main effect of diagnosis on the set shifting or executive function task (the Stroop Test interference score) with the VWS subjects scoring significantly lower than the controls. There was also a main effect of diagnosis for the Rey-Osterreith test of visual perceptual skills. However, in this test, the subjects with VWS scored significantly higher than controls on the immediate and delayed memory portion of the test.

The only cognitive test to have a significant sex by diagnosis interaction was the verbal fluency task. Table 6 shows the results of this task broken down by group and gender. Similar to the findings in FSIQ and VIQ, there was a significant difference between the VWS male subjects compared with the control males, with the VWS subjects scoring significantly lower than normals. However, there was no difference in performance when comparing the female VWS subjects with the female controls.

\section{DISCUSSION}

The pattern of cognitive performance in this adult sample of subjects with VWS is the same pattern seen in both children and adults with isolated cleft of the lip and/or palate (ICLP). 6,8 Several studies of school-age children with ICLP have documented mild generalized cognitive deficits (lower IQ) with specific deficits in language function. ${ }^{18-22}$ Even more compelling are the similarities of performance between the current VWS study group and a larger group of adult males with ICLP examined previously in our lab. ${ }^{8}$ All the tests used for the current study were also used for the previous study of adult males with ICLP. The adult males with ICLP showed significantly lower FSIQ, PIQ, and VIQ scores compared with controls. After controlling for FSIQ, adult males with ICLP also showed specific deficits in verbal fluency and executive function. However, their visual spatial skills as measured by the Rey-Osterreith Complex Figure Test were not below normal and in fact were superior to controls, although this did not reach statistical

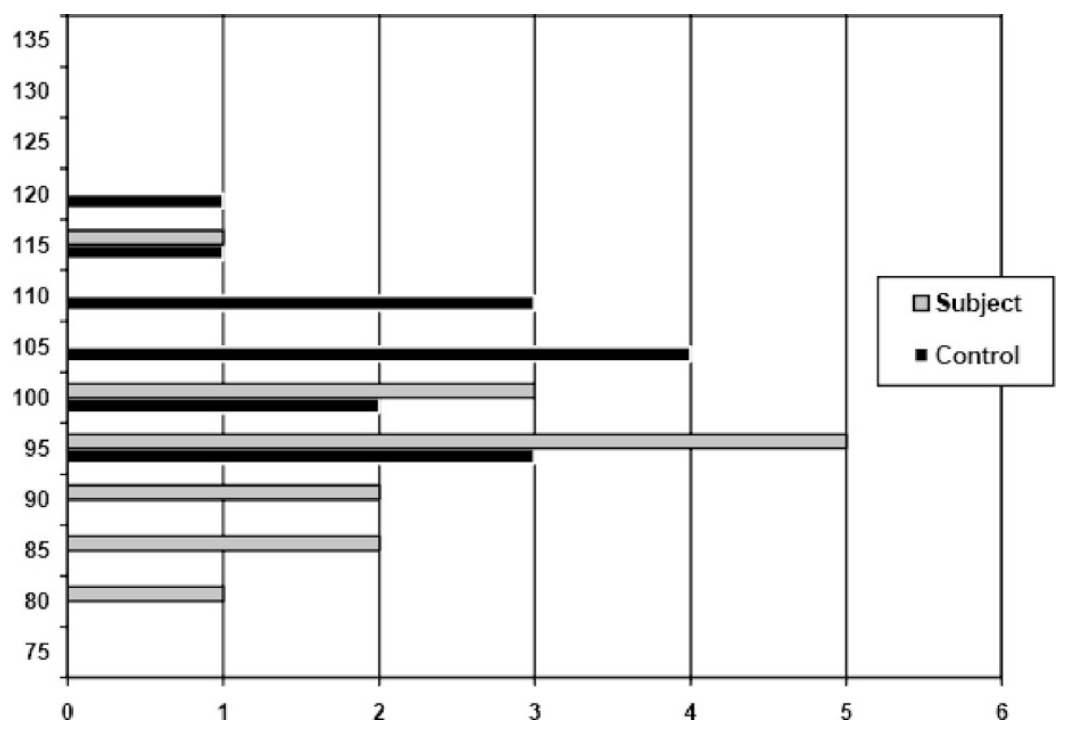

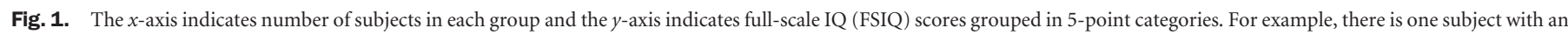
FSIQ between 75 and 80 and five subjects with an FSIQ between 95 and 100 . 
Table 4

Means, SDs, and analysis results of IQ tests by gender

\begin{tabular}{|c|c|c|c|c|c|c|c|c|}
\hline & \multicolumn{2}{|c|}{ Males } & \multicolumn{2}{|c|}{ Post hoc $t$ test } & \multicolumn{2}{|c|}{ Females } & \multicolumn{2}{|c|}{ Post hoc $t$ test } \\
\hline & VWS & Controls & $t$ & $P$ & VWS & Controls & $t$ & $P$ \\
\hline FSIQ & $94.2(6.66)$ & $110.4(8.51)$ & 4.14 & 0.0004 & $100.5(9.69)$ & $102.2(4.85)$ & 0.402 & 0.690 \\
\hline VIQ & $92.0(5.94)$ & $110.5(9.60)$ & 3.98 & 0.0006 & $98.2(11.64)$ & $97.8(6.90)$ & -0.067 & 0.946 \\
\hline PIQ & $99.5(9.21)$ & $108.1(10.17)$ & 1.50 & 0.147 & $104.9(9.82)$ & $109.9(13.2)$ & 0.806 & 0.428 \\
\hline
\end{tabular}

VWS, Van der Woude syndrome; FSIQ, full-scale IQ; VIQ, Verbal IQ; PIQ, Performance IQ.

Table 5

Results of additional cognitive tests across study groups: means (SDs)

\begin{tabular}{|c|c|c|c|c|c|c|}
\hline & \multirow[b]{2}{*}{ Subjects with VWS } & \multirow[b]{2}{*}{ Healthy controls } & \multicolumn{2}{|c|}{ Effect of diagnosis } & \multicolumn{2}{|c|}{ Sex by diagnosis interaction } \\
\hline & & & $F$ & $P$ & $F$ & $P$ \\
\hline Verbal fluency & $41.0(11.38)$ & $45.5(9.05)$ & 1.14 & 0.296 & 5.40 & 0.029 \\
\hline \multicolumn{7}{|l|}{ Verbal memory } \\
\hline Immediate & $53.7(11.50)$ & $60.9(11.98)$ & 1.76 & 0.199 & 0.38 & 0.546 \\
\hline Delayed & $8.68(2.70)$ & $9.12(3.64)$ & 0.23 & 0.634 & 0.18 & 0.674 \\
\hline \multicolumn{7}{|l|}{ Set shifting } \\
\hline Interference score & $42.6(9.54)$ & $51.1(8.25)$ & 6.10 & 0.021 & 0.00 & 0.9569 \\
\hline \multicolumn{7}{|c|}{ Visual perception/memory } \\
\hline Copy & $34.8(1.62)$ & $35.5(0.75)$ & 1.69 & 0.207 & 0.22 & 0.646 \\
\hline Immediate & $23.3(5.70)$ & $18.1(6.61)$ & 6.22 & 0.020 & 0.53 & 0.474 \\
\hline Delayed & $22.3(5.42)$ & $17.7(6.30)$ & 4.67 & 0.041 & 0.40 & 0.532 \\
\hline
\end{tabular}

VWS, Van der Woude syndrome.

Table 6

Results of additional cognitive tests across study groups and sex: means (SDs)

\begin{tabular}{|c|c|c|c|c|c|c|c|c|}
\hline & \multicolumn{2}{|c|}{ Males } & \multicolumn{2}{|c|}{ Post hoc $t$ test } & \multicolumn{2}{|c|}{ Females } & \multicolumn{2}{|c|}{ Post hoc $t$ test } \\
\hline & VWS & Controls & $t$ & $P$ & VWS & Controls & $t$ & $P$ \\
\hline Verbal fluency & $34.1(6.47)$ & $46.8(8.62)$ & 2.08 & 0.048 & $47.9(10.25)$ & $44.0(9.32)$ & -0.811 & 0.425 \\
\hline
\end{tabular}

VWS, Van der Woude syndrome.

significance. In the current study, males with VWS showed a similar pattern with a generalized deficit in FSIQ and VIQ and, after controlling for FSIQ, deficits in verbal fluency and executive function yet superior performance in the visual spatial test.

The current study indicates that adults with VWS have a specific pattern of cognitive deficits, and this deficit is modified substantially by gender. Males with VWS, in general, manifested greater cognitive deficits than females with VWS. Specifically, males with VWS had significantly lower FSIQ and VIQ scores compared with healthy controls. In contrast, females with VWS showed no deficit compared with controls in the IQ measures. After controlling for FSIQ, males with VWS had further specific deficits in both verbal fluency and executive functioning, whereas females with VWS had deficits only in the executive functioning task.

Importantly, the individuals with VWS also showed superior performance in a visual perceptual task. However, the analysis of this test controlled for FSIQ; therefore, the performance on this test is relative to overall intelligence. For the males with VWS, the raw scores of this test do not indicate superior performance over controls. In fact, the raw scores show they perform worse than controls (VWS males mean immediate recall score $=20.5$, control males mean immediate recall score $=24.0$; VWS males mean delayed recall score $=$ 19.8, control males mean delayed recall score $=22.8$ ). Because the males with VWS had significantly lower FSIQ scores, it was only after adjusting for IQ that the visual spatial scores measured higher in the VWS subjects compared with controls. Therefore, the visual spatial skills in VWS males are a relative cognitive strength and not a supernormal skill. For women with VWS, the raw scores for the visual spatial test are substantially higher than the controls, and because they do not have lower FSIQ than their matched normal sample, their adjusted scores remain higher than controls. It may therefore be best to characterize the male VWS cognitive pattern as having gener- 
alized deficit with specific dysfunction of verbal fluency and executive function with sparing of visual spatial skills. Conversely, females with VWS have no generalized cognitive deficits but isolated weaknesses in executive tasks accompanied by strengths in visual spatial skills.

One important aspect of the current study is that although the males with VWS had a significantly lower FSIQ than controls, the mean score was 94.2 , a value not significantly different from the normative mean of 100 per the WAIS-R. However, the normative mean of the WAIS-R is based on a population devised to represent the entire U.S. population. WAIS-R normative data do not account for variance in intelligence due to factors such as age, sex, race, geographic region, and socioeconomic status. The current study is a case-control study in which the subjects are matched by age and gender. Both the VWS and the control samples are of the same age, and both grew up in the Midwest. Although the male control mean IQ of 110 may seem unusually high, it is a score that has been well documented to be specific to this region. ${ }^{17}$ Given this information, the subjects scored significantly lower than would be expected based on their demographic profile.

One potential confound was that the groups were not matched on was parental socioeconomic status. Social class can potentially impinge on brain development through factors such as nutrition, psychological environment, infection, and access to preventive and acute medical care. As a proxy measure to social class, we measured combined years of parental education, and this measure was found to be higher in the controls compared with the VWS group. Therefore, this measure was controlled for in our analysis, and thus the differences in the IQ measures between the males in the study groups are not likely due to differences in parental social class. Conversely, because VWS is an autosomal dominant disorder, one parent of each subject will be affected except in occasional cases of de novo mutations (none in this study). Therefore, the affected parent may well have cognitive deficits (especially if it is the father who is affected), and this may account for the lower education level seen in the parents of the VWS group.

\section{Etiology}

What is the etiology of these deficits? Cognitive abnormalities in ICLP have been hypothesized to be "secondary" to factors such as hearing and/or speech deficits or even to the social effects of facial disfigurement. ${ }^{23,24}$ However, work from our group (see below) and others ${ }^{25,26}$ is mounting evidence to support the theory that the brain structure/function abnormalities in orofacial clefting disorders are in fact a primary brain problem due to aberrant neural development. From a series of studies on adult males with ICLP, our laboratory has reported an increased incidence of developmental brain anomalies ${ }^{27}$ and a structural brain pattern of abnormally increased volumes of the anterior cerebrum (frontal and parietal lobes) with accompanying reduction in volumes of the posterior cerebrum (temporal and occipital lobes) and cerebellum..$^{28,29}$ Moreover, these structural deficits were directly related to cognitive dysfunction. These findings have added substantial support to the no- tion that the cognitive deficits associated with ICLP are primarily due to abnormal brain structure and that, like the oral cleft, the abnormality in brain structure and function is due to aberrant development.

\section{Further support for a neurodevelopmental etiology: the gender effect}

Females with VWS fared substantially better than males with VWS in regard to cognitive performance, again supporting our hypothesis that there would be a gender effect in cognitive function. This finding is similar to the findings reported by Richman and Eliason ${ }^{22}$ of lower VIQ in males with ICLP. In addition, it is a well-known phenomenon that all neurobehavioral developmental disorders (autism, mental retardation, attention-deficit/hyperactivity disorder) are more common and/or more severe in males. ${ }^{910}$ This gender effect is particularly true for developmental learning disorders specific to speech and language. VWS is an autosomal dominant disorder and the prevalence of VWS is equal between men and women. However, VWS is a developmental disorder; the craniofacial manifestations of oral clefting and/or lip pits are present at birth and caused by abnormal development. The fact that there is an expected gender effect on the brain structure/function profile of subjects with VWS supports the theory that these deficits are developmental in origin.

The current study has limitations that should be discussed, including small sample size. Without doubt, these findings need to be replicated in a separate and larger sample of subjects with VWS. Another limitation is that $50 \%$ of the VWS subjects were all part of one extended pedigree. Because IQ is highly heritable $^{30}$, it is possible that the shared genes of the family influenced test performance and that the differences in IQ between the two groups is driven by this factor, independent of VWS. The seven family members come from four separate nuclear families within the pedigree. Although the sample size becomes exceedingly small, when the VWS is limited to the remaining seven subjects who are not related to each other (the seven VWS from the same pedigree now eliminated), there is no overall effect of diagnosis on FSIQ $(F=2.72, P=0.113)$, yet there is a significant sex by diagnosis interaction $(F=7.38, P=$ $0.015)$. When this small sample is broken down by men $(n=4$ VWS, $n=7$ controls) and women ( $n=3$ VWS, $n=7$ controls), the males with VWS have a significantly lower FSIQ compared with the control males $(t=3.03, P=0.007)$, whereas there is no difference in IQ comparing the women in each sample $(t=2.00, P=0.327)$, the same pattern of results seen in the larger sample. This post hoc analysis confirms that the findings of the total sample of VWS $(N=14)$ is not confounded by the shared genes of the seven members from the same extended family and reflects instead the cognitive dysfunction of VWS.

\section{ACKNOWLEDGMENTS}

This research was funded by a Mentored, Patient Oriented Career Development Award 5 K23 DE000417-05. The control 
data were collected through support of the following $\mathrm{NIH}$ grants: MH60990, MH40856, MH031593 to N.C. Andreasen.

\section{References}

1. Kondo S, Schutte BC, Richardson RJ, Bjork BC, et al. Mutations in IRF6 cause Van der Woude and popliteal pterygium syndromes. Nat Genet 2002;32:285-289.

2. Rizos M, Spyropoulos MN. Van der Woude syndrome: a review. Cardinal signs, epidemiology, associated features, differential diagnosis, expressivity, genetic counselling and treatment. Eur J Orthod 2004;26:17-24.

3. Salamone FN, Myer CM 3rd. Van der Woude syndrome: the most common cleft syndrome. Otolaryngol Head Neck Surg 2004;131:141.

4. Kantaputra PN, Sumitsawan Y, Schutte BC, Tochareontanaphol C. Van der Woude syndrome with sensorineural hearing loss, large craniofacial sinuses, dental pulp stones, and minor limb anomalies: report of a four-generation Thai family. Am J Med Genet 2002;108:275-280.

5. Zucchero TM, Cooper ME, Maher BS, Daack-Hirsch S, et al. Interferon regulatory factor 6 (IRF6) gene variants and the risk of isolated cleft lip or palate. $\mathrm{N}$ Engl J Med 2004;351:769-780.

6. Eliason M. Neuropsychological perspectives of cleft lip and palate. In: Bardach J, Morris H, editors. Multidisciplinary management of cleft lip and palate. Philadelphia: WB Saunders, 1990:825-831.

7. Richman LC, Wilgenbusch T, Hall T. Spontaneous verbal labeling: visual memory and reading ability in children with cleft. Cleft Palate Craniofac J 2005;42:565-569.

8. Nopoulos P, Berg S, VanDemark D, Richman L. Canady J, Andreasen NC. Cognitive dysfunction in adult males with non-syndromic clefts of the lip and/or palate. Neuropsychologia 2002;40:2178-2184.

9. American Psychiatric Association. Diagnostic and Statistical Manual of Mental Disorders, Fourth Edition. 1994, Washington, DC: American Psychiatric Association. 37-121.

10. Castle D, Murray R. The neurodevelopmental basis of sex differences in schizophrenia. Psychol Med 1991;21:565-575.

11. Wechsler D. Wechsler Adult Intelligence Scale-Revised, Manual. Cleveland, OH: The Psychological Corporation, 1981.

12. Benton AA, Hamsher K. Multilingual aphasia examination. Iowa City, IA: The University of Iowa Press, 1976.

13. Rey A. L'examen clinique en psychologie. Paris, France: Presses Universitaires de France, 1964.

14. Osterreith P. Le test de copie d'une figure complexe. Arch Psychol 1944;30:206-356.
15. Rey A. L'examen psychologique dans les cas d'encephalopathie traumatique. Arch Psychol 1941;28:286-340.

16. Golden C. Stroop Color and Word Test: a manual for clinical and experimental uses. Chicago: Stoelting, 1978.

17. Lindgren S, DeRenzi E, Richman L. Cross national comparisons of developmental dyslexia in Italy and the United States. Child Dev 1985;56:1404-1417.

18. Eliason MJ, Richman LC. Language development in preschoolers with cleft. Dev Neuropsychol 1990;6:173-182.

19. Kommers M, Sullivan M. Written language skills of children with cleft palate. Cleft Palate J 1979;16:81-85.

20. Richman L, Eliason M. Psychological characteristics of cleft lip and palate children: intellectual achievement, behavior and personality variables. Cleft Palate Craniofac J 1982;19:249-257.

21. Richman LC. Cognitive patterns and learning disabilities in cleft palate children with verbal deficits. J Speech Hear Res 1980;23:447-456.

22. Richman LC, Eliason M. Disorders of communication, developmental language disorders and cleft palate. In: Walker CE, Roberts MC, Editors. Handbook of child clinical psychology-revised. New York: John Wiley \& Sons, 2001.

23. Estes R, Morris H. Relationship among intelligence, speech proficiency, and hearing sensitivity in children with cleft palates. Cleft Palate J 1970;7:763-773.

24. Sak RJ, Ruben RJ. Effects of recurrent middle ear effusion in preschool years on language and learning. J Dev Behav Pediatr 1982;3:7-11.

25. Ceponiene R, Hukki J, Cheour M, Haapanen MC, Ranta R, Naatanen R. Cortical auditory dysfunction in children with oral clefts: relation with cleft type. Clin Neurophysiol 1999;110:1921-1926.

26. Nagore E, Sanchez-Motilla JM, Febrer MI, Serrano G, Bonilla J. Aliaga A. Congenital lower lip pits (Van der Woude syndrome): presentation of 10 cases. Pediatr Dermatol 1998; 15:443-445.

27. Nopoulos P, Berg S, Van Demark D, Richman L, Canady J, Andreasen NC. Increased incidence of a midline brain anomaly in adult males with nonsyndromic clefts of the lip and/or palate. J Neuroimaging 2001;11:418-424.

28. Nopoulos P, Berg S, Canady J, Richman L, Van Demark D, Andreasen NC. Abnormal brain morphology in adult males with isolated clefts of the lip and/or palate: a preliminary analysis. Cleft Palate Craniofac J 2000;37:441-446.

29. Nopoulos P, Berg S, Canady J, Richman L, Van Demark D, Andreasen NC. Structural brain abnormalities in adult males with clefts of the lip and/or palate. Genet Med 2002;4:1-9.

30. Devlin B, Daniels M, Roeder K. The heritability of IQ. Nature 1997;388:468-471. 